\title{
Evans' Argument and Vague Objects
}

\author{
Graham Priest \\ Philosophy Programs, CUNY Graduate Center and the University of Melbourne
}

\begin{abstract}
In 1978, Gareth Evans published a short and somewhat cryptic article purporting to establish that there are no vague objects. This paper is a commentary on this. Prima facie, the claim that there are no vague objects is clearly false. Mt Everest, for example, has no precise boundaries. And if this is so, there must be something wrong with Evans' argument. In the paper, I discuss what this is, giving a model of vague objects in the process.
\end{abstract}

\section{Introduction}

In 1978, Gareth Evans published a short and somewhat cryptic article purporting to establish that there are no vague objects. ${ }^{1}$ The paper generated a small literature. ${ }^{2}$ This essay is a somewhat belated contribution to it.

Prima facie, the claim that there are no vague objects is clearly false. None of the following has precise boundaries: Mt. Everest, the Coral Sea, the European feudal epoch, the period of Medieval philosophy. ${ }^{3}$ And if this is so, there must be something wrong with Evans' argument. In what follows, I will give a model of vague objects, and so diagnose what is wrong with it.

\footnotetext{
${ }^{1}$ Evans (1979).

${ }^{2}$ For an introduction to this, see Keefe and Smith (1996), pp. 52-56.

${ }^{3}$ In this essay, I do not argue that there really are vague objects in re. But these examples certainly seem to be so: their boundaries in space and time are indeterminate. So they at least put the onus of proof on those who claim that they are not. The main point of the paper is to argue that Evans' argument does not discharge this onus. Could there be vague objects other than those with spatio-temporal boundaries? Perhaps. Maybe some abstract objects, such as symphonies, proofs, and even essays, such as this.
}

Australasian Journal of Logic (18:3) 2021, Article no. 1 


\section{Evans' Argument}

Let us start with Evans' argument. First, some terminology. Let us use the following symbols with the given glosses:

- $\square A:=$ It is determinately true that $A$

- $\Delta A:=\square A \vee \square \neg A$ (It is determinate whether $A$ )

- $\nabla A:=\neg \Delta A=\neg \square A \wedge \neg \square \neg A$ (It is indeterminate whether $A)^{4}$

Evans' argument now goes as follows: ${ }^{5}$

- Suppose that $\nabla a=b$

- $\Delta a=a($ since $\square a=a)$

- So $\neg \nabla a=a$

- So $a \neq b$

It ends there. This leaves us with two puzzles. The first is what, exactly, we are supposed to have proved. This argument is clearly meant to be some kind of reductio of the supposition. So Evans appears to be assuming that $a \neq b \rightarrow \neg \nabla a=b{ }^{6}$ But why should we suppose this? This is the claim that $a \neq b \rightarrow \square a=b \vee \square a \neq b$. If it is false that $a=b$ then it cannot be the case that $a=b$ is determinately true; so presumably we have to assume that $a \neq b \rightarrow \square a \neq b$. This isn't exactly the question-begging $a=b \rightarrow \square a=b$, but the two would seem to stand or fall together. Moreover, there are accounts of vagueness where the principle $A \rightarrow \square A$ fails quite generally. ${ }^{7}$

Evans gestures at a way of bringing the argument to a formal contradiction, by assuming that the logic of $\square$ is S5. The argument is not spelled out, but presumably it is something like the following. We have just shown that:

\footnotetext{
${ }^{4}$ Evans calls $\nabla$ the dual of $\Delta$, but the terminology is somewhat aberrant. It would be normal to call $\neg \Delta \neg$ the dual of $\Delta$. $\nabla$ is simply the negation.

says that $\Delta$ and $\nabla$ are duals. This is not correct. The dual of $\Delta A$ is $\neg \Delta \neg A$, not $\nabla A$. However, if we assume the law of double negation, $\neg \Delta \neg A$ is equivalent to $\nabla A$.

${ }^{5}$ This is a slightly simplified version. Evans' actual argument is more complicated, using lambda abstractions. For reasons I will explain in fn. 13, the extra complication buys nothing.

${ }^{6}$ Note that one cannot simply infer $\square a \neq b$ (and so $\neg \nabla a=b$ ) from $a \neq b$ by Necessitation, since the argument for $a \neq b$ depends on an assumption.

${ }^{7}$ E.g., that of Field (2003).
} 
- $\nabla a=b \models a \neq b$

- So $\square \nabla a=b \models \square a \neq b$

- In $\mathrm{S} 5^{8} \nabla a=b=\square \nabla a=b$

- So $\nabla a=b=\square a \neq b$

- But $\square a \neq b \models \Delta a=b$

- That is, $\square a \neq b=\neg \nabla a=b$

- So $\nabla a=b \mid=\neg \nabla a=b$

- Hence $\models \neg \nabla a=b$

There are grounds to doubt that the logic of $\square$ is $S 5$. We will come to these in due course. For the moment, let us set this matter aside.

The second problem is this. Let us assume that we have shown that $\neg \nabla a=b$, that is, $\Delta a=b$ : all identities are determinate. How does that show that there are no vague objects? Presumably the thought is that vague objects will give rise to indeterminate identities. But this is not at all obvious. We might say that two objects are the same if any way of precisifying one precisifies the other; and two objects are distinct if there is a way of precisifying one but not the other. (A precise object has, by definition, only one precisification.) Thus, Australia is not New Holland $;^{9}$ but Sagarmāthā is Chomolungma. ${ }^{10}$ Such identities are determinate, since they are not hostage to the vicissitudes of precisification. Hence, with such truth conditions, vague objects do not give rise to indeterminate identities. However, let us set this matter aside for the moment also.

For as is clear, the core of the argument itself is simply an application of the distinctness of discernibles:

$$
\text { - } A_{x}(a), \neg A_{x}(b) \models a \neq b
$$

where, note, $A$ contains $\nabla$. This is just a contraposed version of the indiscernibility of identicals (SI): $a=b, A_{x}(a) \models A_{x}(b)$. So when all the frills

\footnotetext{
${ }^{8} \nabla A \mid=\neg \square A \wedge \neg \square \neg A$. By $S 5$ principles, $\nabla A=\square \neg \square A \wedge \square \neg \square \neg A$. So $\nabla A=\square(\neg \square A \wedge$ $\neg \square \neg A)$. That is, $\nabla a=b \models \square \nabla a=b$.

${ }^{9}$ 'New Holland' is the name that early European explorers called the Australian land mass. At the time, the existence of Tasmania was unknown.

${ }^{10}$ When the Nepalese gazed at the peak which Europeans were later to call Mt Everest, they called it Sagarmāthā. When the Tibetans gazed at the same peak from the other side of the Himalayas, the called it Chomolungma.
}

Australasian Journal of Logic (18:3) 2021, Article no. 1 
are stripped off, this is just a determinacy analogue of an old modal friend: the necessity of identities: $a=b=\square a=b$ (since $\square a=a) .{ }^{11}$ This principle fails in modal logics of so called contingent identity, ${ }^{12}$ which will therefore invalidate Evans' argument. Let us see how. ${ }^{13}$

\section{Contingent Identity}

Actually, it's not clear that the determinacy- $\square$ is a modal operator. There are certainly accounts of vagueness in which borderline cases are neither true nor false. ${ }^{14}$ So, $\square A$ is true iff $A$ is true; $\Delta A$ is true iff $A$ is either true or false; and $\nabla A$ is true iff $A$ is neither true nor false. Hence the inference $A \vdash \square A$ is valid, and $\square$ is not modal operator.

What happens to Evans' argument in this case? If the logic is something like Strong Kleene ${ }^{15}$ and identity behaves normally, $a=b, \Delta a=a \models \Delta a=$ $b$. (The second premise is, in fact, redundant.) However, the contraposed form fails: $\neg \Delta a=b, \Delta a=a \models a \neq b$. (If $a=b$ is neither true nor false, the premises are true, but not the conclusion.) So Evans' argument fails because of this. ${ }^{16}$

But set all this aside as well, and let us assume that the determinacy- $\square$ is a modal operator, and give it a world-semantics.

\footnotetext{
${ }^{11}$ See, e.g., Kripke (1971).

${ }^{12}$ See, e.g., Priest (2008), ch. 17. This contains further details of the semantics of the next section. There, I simply identity an element in the domain with its precisifying function.

${ }^{13}$ There is one frill of Evans' argument on which I have not so far commented. Evans' actual formulation of the argument employs $\lambda$-terms and $\lambda$-conversion, moving from $\nabla a=$ $b$ to $[\lambda x \nabla a=x] b$, and $\neg \nabla a=a$ to $\neg[\lambda x \nabla a=x] a$, before applying the distinctness of discernibles. This just hides the fact that what is at issue is simply whether, if $a=b$, the first can be substituted for the second in modal contexts. To understand why, one needs to think about the semantics of $\lambda$-terms. A simple approach to these is to take the extension of the predicate $[\lambda y A(y)] x$ (or the property which it denotes) at world $w$ to be the extension of the formula $A(x)$ at $w$. Given this, $\lambda$-conversion is certainly satisfied. But just because of this coextensionality, if SI fails for $\square x=a$, it fails equally for $[\lambda y \square y=a] x$. Of course, this may fairly be taken to show that predication by the $\lambda$-term is not de re predication, as Evans surely intended. To ensure that it is de re predication, the extension of $[\lambda y A(y)] x$ at world $w$ has to be (equivalent to) $\exists y(y=x \wedge A(y))$ at $w$ - the reference being obtained by the external scope quantifier. But now $\lambda$-conversion in one direction (the one invoked in contraposed form in the argument) presupposes SI. For $[\lambda x A(x)] a$ then entails $\exists y(y=a \wedge A(y))$; and SI is required to move to $A(a)$.

${ }^{14}$ E.g., Tye (1994).

${ }^{15}$ See Priest (2008), ch. 7.

${ }^{16}$ See Priest (2008), 21.9.
}

Australasian Journal of Logic (18:3) 2021, Article no. 1 
Take a first-order language with identity, $\square$, and, for simplicity, no function symbols. We may think of $\vee, \rightarrow, \exists, \diamond$ as defined in the usual way, and $\Delta$ and $\nabla$ as defined as above.

An interpretation is a structure $\langle W, R, D, H, \pi, \delta\rangle . W$ is a set of "worlds". Intuitively, these are situations at which we arrive when we precisify things. $R$ is the universal accessibility relation. That is: for all $w, w^{\prime} \in W, w R w^{\prime}$.

$H$ is a certain domain of objects. $D$ is the domain of quantification. For every $d \in D, \pi(d)$ is a map from $W$ to $H$. Intuitively, it specifies the precisification of $d$ at $w$. I will write $\pi(d)(w)$ as $|d|_{w}{ }^{17}$

If $c$ is any constant, $\delta(c) \in D$, and if $P_{n}$ is any $n$-place predicate symbol, $\delta\left(P_{n}\right)$ is a function from worlds to a subset of $H^{n}$. Let us write $\delta\left(P_{n}\right)(w)$ as $\delta_{w}\left(P_{n}\right)$. One may think of this as the precisification of $P_{n}$ at $w{ }^{18}$

The truth conditions for atomic sentences are: ${ }^{19}$

- $w \Vdash P c_{1} \ldots c_{n}$ iff $\left\langle\left|\delta\left(c_{1}\right)\right|_{w}, \ldots,\left|\delta\left(c_{n}\right)\right|_{w}\right\rangle \in \delta_{w}\left(P_{n}\right)$

If the extension of a predicate is the same at all worlds of an interpretation, it has only one precisification, and so is precise. The extension of identity is given, as is to be expected, by: $\delta_{w}(=)=\{\langle h, h\rangle: h \in H\}$. Hence, identity is a precise predicate.

The truth conditions for the logical operators are as usual in $S 5$. For the quantifiers, we assume that the language has been augmented with a constant, $c_{d}$, for every $d \in D$, such that $\delta\left(c_{d}\right)=d$. Then we have:

- $w \Vdash \neg A$ iff $w \Vdash 4$

- $w \Vdash A \wedge B$ iff $w \Vdash A$ and $w \Vdash B$

- $w \Vdash \forall x A$ iff for all $d \in D, w \Vdash A_{x}\left(c_{d}\right)$

- $w \Vdash \square A$ iff for all $w^{\prime} \in W$, such that $w R w^{\prime}, w^{\prime} \Vdash A$

\footnotetext{
${ }^{17}$ As a referee observed, one could simply take $H$ to be $\left\{h: \exists d \in D \exists w \in W h=|d|_{w}\right\}$. Members of $H$ not of this form have no effect on the semantics.

${ }^{18}$ I note that if we wished, we could introduce a domain of properties and relations, and let $\delta$ map each $n$-place predicate to an $n$-place relation. $\pi$ could then map each member of that domain to its precisification at world $w$. What I now write as $\delta_{w}\left(P_{n}\right)$ would then be $\left|\delta\left(R_{n}\right)\right|_{w}$, where $R_{n}$ is the $n$-ary relation referred to by $P_{n}$. This would give us a theory of vague properties. However, this is not required here, since the language is first-order.

${ }^{19}$ Perhaps the place where one is most likely to meet contingent identity semantics is in connection with "4-dimensionalism". There, the indices of evaluation are times, $t$, and an object, $d$, is a 4-dimensional "worm". $|d|_{t}$ is the temporal part of $d$ at $t$. Then, for example, 'Socrates is sitting' is true at time $t$ iff the temporal part of Socrates at time $t$ is in the extension of 'is sitting' at time $t$.
}

Australasian Journal of Logic (18:3) 2021, Article no. 1 
Validity is defined as truth preservation at all worlds of all interpretations.

Ignoring identity, the modal logic is that of constant-domain $S 5 .^{20}$ For identity, a simple induction, left as an exercise, proves that if $A$ contains no modal operators, $a=b, A_{x}(a) \models A_{x}(b)$. However, SI fails in modal contexts: $a=b, \square a=a \not \models \square a=b . \square a=a$ is true at every world, so we may ignore this. As a counter-example, take an interpretation, with worlds, $w$ and $w^{\prime}$, such that $|\delta(a)|_{w}=|\delta(b)|_{w}$ but $|\delta(a)|_{w^{\prime}} \neq|\delta(b)|_{w^{\prime}}$. Then $a=b$ is true at $w$, but false at $w^{\prime}$. Hence $\square a=b$ is false at $w$.

In particular, then, Evans' argument fails. Indeed, there are interpretations where $\nabla a=b$, that is, $\neg \square a=b \wedge \neg \square \neg a=b$, that is, $\diamond a \neq b \wedge \diamond a=b$, is true at some worlds. The interpretation just described is one such. ${ }^{21}$

\section{Global Predicates}

Though SI fails in the contingent-identity semantics, it is possible to formulate an enthymematic version. Let us add a new monadic predicate, $\mathbb{C}$, to the language. Intuitively, $\mathbb{C} x$ expresses the fact that $\pi(x)$ is a constant function. One may take it to express the thought that $x$ is a precise object, since it has only one precisification.

The truth conditions of the predicate are as follows:

- $w \Vdash \mathbb{C} c$ iff for all $w_{1}, w_{2} \in W,|\delta(c)|_{w_{1}}=|\delta(c)|_{w_{2}}$

It is easy to check that if $\mathbb{C} a, \mathbb{C} b$, and $a=b$, are true at a world of some interpretation, then $a=b$ is true at every world. It follows easily that

\footnotetext{
${ }^{20}$ See Priest (2008), ch. 14.

${ }^{21}$ In an interpretation there can be distinct members of $D$ with the same set of precisifications. It is very natural to suppose that the objects in question are really the same. This can be imposed as an extra constraint on interpretations. Specifically, consider the equivalence relation, $\sim$, on members of $D . d_{1} \sim d_{2}$ iff $\forall w_{1} \exists w_{2}\left|d_{1}\right|_{w_{1}}=\left|d_{2}\right|_{w_{2}}$ and $\forall w_{2} \exists w_{1}\left|d_{2}\right|_{w_{2}}=\left|d_{1}\right|_{w_{1}}$. Then consider the constraint, $C$ : for all $d_{1}, d_{2} \in D$ (if $d_{1} \sim d_{2}$ then $d_{1}=d_{2}$ ). Nothing in this paper changes if we we require all interpretations to satisfy constraint $C$. In particular, the counter-model just given can be found in such an interpretation.

In fact, adding the constraint does not change the consequence relation. If truth is preserved in all interpretations, it is preserved in all constrained interpretations. Conversely, if something fails in an interpretation, it fails in a constrained interpretation. To see this, take any interpretation, and for every $d \in D$, add a new member, $h_{d}$, to $H$. Then for every $d \in D$, choose some world, $w$. Let $|d|_{w}$ be $h$. Then extend the extension of every predicate with the $n$-tuple which can be obtained by replacing every occurrence of $h$ with $h_{d}$. Finally, change $\pi$ so that $|d|_{w}$ is not $h$, but $h_{d}$. In the modified interpretation every member of $D$ has a precisification that no other member of $D$ has. So condition $C$ is satisfied. But a simple induction shows that the same things are true in the original interpretation and the modified interpretation.
}

Australasian Journal of Logic (18:3) 2021, Article no. 1 
$\mathbb{C} a, \mathbb{C} b, a=b \mid=\square a=b$, and, more generally, that $\mathbb{C} a, \mathbb{C} b, a=b, A_{x}(a) \models$ $A_{x}(b)$, for arbitrary $A$.

In $\S 2$ I noted that vague objects do note necessarily give rise to indeterminate identities. We can now make that thought precise, since $\Delta a=b \forall \mathbb{C} a$. (Let $a$ and $b$ denote the same non-constant function.)

$=$ is not the notion of identity given in $\S 2$. However, one may add yet another global predicate to the language, $\equiv$, which expresses this. ${ }^{22}$ One might think of this as identity simpiciter, as opposed to identity-at-a-world.

- $w \Vdash c_{1} \equiv c_{2}$ iff $\delta\left(c_{1}\right)=\delta\left(c_{2}\right)$

If $w \Vdash c_{1} \equiv c_{2}$ then, for all $w^{\prime},\left|\delta\left(c_{1}\right)\right|_{w^{\prime}}=\left|\delta\left(c_{2}\right)\right|_{w^{\prime}}$. A simple induction (left as an exercise) shows that $a \equiv b, A_{x}(a) \mid=A_{x}(b)$, and, in particular, that $a \equiv b \models \square a \equiv b$. Moreover, $a \not \equiv b \mid \square a \not \equiv b$. Thus, there are no indeterminate identities of this kind: $\models \Delta a \equiv b$.

Given Evans' ruminations, it might be thought that this shows that there are no vague objects, but this is not so. The determinacy of this notion of identity notwithstanding, it remains the case that some members of the domain have a single precisification, and so are precise, and some of them have more than one, and so are vague. In particular, it is still the case that $\Delta a \equiv b \not \models \mathbb{C} a$. (Take $\delta(a)$ and $\delta(b)$ to be the same, $\pi(\delta(a))$ to be a non-constant function.)

Indeed, there is just no way to rescue the argument whatever one does, simply because there are models with vague objects; that is, $d$ s for which $\pi(d)$ is not constant.

\section{$5 \quad$ Truth Simpliciter}

The semantics of $\S 3$ define truth-with-respect-to-a-precisification; but what about truth simpliciter? What does it mean to say Mt Everest is in the Himalayas (period)? A natural suggestion is that this is true iff it is true with respect to all precisifications, i.e., determinately true. If $\Sigma$ is a set of sentences, let $\square \Sigma=\{\square A: A \in \Sigma\}$. The consequence relation for truth preservation, $\models \square$, may then be defined thus: $\Sigma \mid=\square A$ is $\square \Sigma \mid=\square A$. ${ }^{23}$

\footnotetext{
${ }^{22}$ Ordinary predicates of the language may be termed local. To see whether they apply at a world, one need only consult the goings-on at that world. By contrast, to see whether $\mathbb{C}$ and $\equiv$ apply, one has to consult other worlds. We may call such a predicates global. (In this sense, the truth functions are local, but the modal operators are global.)

${ }^{23}$ In an obvious sense, this is a supervaluation semantics; and like all such semantics, it will have non-classical consequences in the multiple-conclusion version of the logic. Thus,
} 
SI fails for $=$ in modal contexts; but as we have seen, $\square a=b, A_{x}(a) \models$ $A_{x}(b)$, for all contexts, $A$. Hence, $a=b, A_{x}(a) \models \square A_{x}(b)$. So it might be thought that the Evans argument goes through using $\models_{\square}$ as the consequence relation. It does not. The reason is that $=_{\square}$ does not contrapose. As is easy to check, $A \models_{\square} \square A$ (that is, $\square A \models \square \square A$ ), but it is not the case that $\neg \square A=\square \neg A$ (that is, $\square \neg \square A=\square \neg A$ ). In particular, even though:

- $[\neg \nabla a=a] a=b \mid,=\square \nabla a=b$

we do not have:

- $[\neg \nabla a=a,] \nabla a=b \models_{\square} a \neq b$

as a simple counter-model shows. ${ }^{24}$ Indeed, it is not the case that $\models_{\square} \neg \nabla a=$ $b$, that is, $\models \square \neg \nabla a=b$. For this would entail that $\models \neg \nabla a=b$, which we know to be false.

A final note on $=_{\square}$. In any normal modal logic, if $\Sigma \models A$ then $\square \Sigma \models \square A$. That is, $\models$ is a subrelation of $\models_{\square}$. In the logic $K$, they are identical. For suppose that $\Sigma \not \models A$. Take a counter-model. Then there is a world, $w$, in this where all the members of $\Sigma$ are true, and $A$ is false. Construct a new interpretation which is the same, except that it adds a world, $w^{\prime}$, which accesses $w$ and only $w$. Then every member of $\square \Sigma$ is true at $w^{\prime}$, and $\square A$ is not. Hence $\Sigma \nvdash_{\square} A$. However, in general, $\models$ is a proper subrelation. Consider the logic $K_{\tau}$. Then $A \not \models \square A$; but $\square A \models \square \square A$. That is, $A \models \square \square A$.

Bounding on the other side, if the logic contains $\square A \rightarrow A$, then $\Sigma \models \square A$, that is $\square \Sigma \models \square A$, entails $\square \Sigma \models A$. In general, this is a proper subrelation. Thus, in $K_{\rho}, \square A \not \models \square \square A$; that is $A \not \models \square \square A$. But $\square A \models \square A$. i.e., $A \models \square$ $A$. However, if the logic also contains $\square A \rightarrow \square \square A$ then the relations are identical. For:

$$
\begin{aligned}
\square \Sigma \mid=A & \Rightarrow \square \square \Sigma \models \square A \\
& \Rightarrow \square \Sigma \models \square A \\
& \Rightarrow \Sigma \mid=\square A
\end{aligned}
$$

$A \vee B \not \models \square A, B$. Another way to define truth simpliciter is truth at some precisification. The consequence relation, $\Sigma \models \diamond A$, is now $\diamond \Sigma \models \diamond A$. This is a sort of subvaluational or discussive logic. (On subvaluations, see Priest (2008) 7.10.5b-7.10.5d. On discussive logic, see Priest (2002), 4.2) This consequence relation has features dual to the supervaluational one. For example, $A, B \not \models \diamond A \wedge B$.

${ }^{24}$ The interpretation at the end of $\S 3$ will do. This makes $\nabla a=b$ true at every world, so $\square \nabla a=b$ holds at every world. But $a \neq b$ fails at some worlds, so $\square a \neq b$ fails at all worlds.

Australasian Journal of Logic (18:3) 2021, Article no. 1 


\section{Higher Order Indeterminacy}

Until now we have assumed (with Evans) that the modal logic of $\square$ is $S 5$. In particular, it validates $\square A \rightarrow \square \square A$ and $\neg \square A \rightarrow \square \neg \square A$, which rule out any indeterminacy in determinacy. It is natural, however, to suppose that there is such a thing as higher order vagueness, so that these principles should not be valid. We can, of course, remove them by moving to a weaker logic, and, in particular, by making the accessibility relation, $R$, weaker. But how to do this in a principled way?

First, we suppose that in any interpretation, each local $n$-place predicate, $P$, comes with inner and outer bounds, $P^{I}$ and $P^{O}$. These mark the limits of how much $P$ may vary when it is precisified. That is, for any world, $w$ :

$$
\text { - } P^{I} \subseteq|\delta(P)|_{w} \subseteq P^{O} \subseteq H^{n}
$$

Such is a natural enough thought. Suppose that $P$ is 'is red'. One can precisify this by drawing the line amongst objects that are borderline red. But the cut must include all clearly red objects: these are all in the inner bound. And it must exclude all the things that are clearly not red (blue, green, etc): these are beyond the outer bound.

Next, note that these limits of precisification, as specified, are crisp subsets of $H^{n}$. They set rigid boundaries to the limits of variation (modulo a given interpretation). This is exactly what higher order vagueness rules out. The bounds of variation are themselves subject to precisification, and so may change from world to world. In other words, we need to assume that the inner and outer bounds are themselves world-dependent. Let us write the bounds of $P$ at world $w$ as $\left|P^{I}\right|_{w}$ and $\left|P^{O}\right|_{w}$. The conditions on worlds and bounds must then be revised accordingly:

$\mathbf{P}:\left|P^{I}\right|_{w} \subseteq|\delta(P)|_{w} \subseteq\left|P^{O}\right|_{w} \subseteq H^{n}$

We may now use the bounds to define an appropriate accessibility relation. Namely, for every $w, w^{\prime} \in W$ :

R: $w R w^{\prime}$ iff for every $P,\left|P^{I}\right|_{w} \subseteq|\delta(P)|_{w^{\prime}} \subseteq\left|P^{O}\right|_{w}$

In other words, the precisification at $w^{\prime}$ is within the bounds set by $w$. If the bounds on precisification set by $w$ are not respected in $w^{\prime}$, then the precisification of $w^{\prime}$ will not be a legitimate one for $w$.

What is going on here, technically, is that the accessibility relation is dropped from an interpretation. Instead, the interpretation comes with a pair of functions, $I$ and $O$, which map every world, $w$, and $n$-place predicate, 
$P$, to a subset of $H^{n}$, such that $\left|P^{I}\right|_{w} \subseteq\left|P^{O}\right|_{w}$. $\delta$ is then constrained by the condition $\mathbf{P}$; and the accessibility relation $R$ is defined by condition $\mathbf{R}$.

Thus defined, $R$ is clearly reflexive (so $=\square A \rightarrow A$ ). But it need not be transitive. For suppose that the range of a monadic predicate is a subset of the reals, and that the inner bound, extension, and outer bound, of some monadic predicate, $P$, are the left-hand sides of Dedekind cuts. Suppose, further, that $P$ is the only predicate relevant in defining $R$. (All the other predicates, we may suppose, satisfy the defining condition of $R$, for all $w$ and $w^{\prime}$. This can be done by making their bounds world-invariant.) Finally, suppose that there are three worlds where the cuts are as follows:

$\begin{array}{cccc} & \text { Inner bound } & \text { Extension } & \text { Outer bound } \\ w_{1} & -2 & 0 & +2 \\ w_{2} & -4 & 1 & +4 \\ w_{3} & -6 & 3 & +6\end{array}$

As is easy to check: $w_{1} R w_{2}, w_{2} R w_{3}$, but it is not the case that $w_{1} R w_{3}$.

Moreover, the same example shows that $R$ need not be Euclidean (that is, it is not the case that if $x R y$ and $x R z$ then $y R z)$. For $w_{2} R w_{1}$ and $w_{2} R w_{3}$, but it is not the case that $w_{1} R w_{3}$.

Hence, given that the accessibility relation, $R$, is defined in this way, the logic does not verify the higher-order-excluding $\square A \rightarrow \square \square A$ and $\neg \square A \rightarrow$ $\square \neg \square A$.

I note that none of this affects the failure of the substitutivity of identicals relevant to the Evans argument. The counter-models we had are, in effect, just special cases, where the bounds may be taken as world-invariant, making $R$ the universal relation. Indeed, moving to the weaker logic provides another reason why Evans' argument fails, since his attempt to fill in the gap of his argument (see $\S 2$ ), uses $S 5$ essentially.

\section{Two Objections}

The vicissitudes of higher order vagueness notwithstanding, the contingent identity semantics gives us a model of vague objects. The objects are the members, $d$, of $D$, and $|d|_{w}$ is a precisification of $d$ in situation $w$. In this final section, I will consider two objections to the effect that these semantics do not really deliver a semantics of vague objects.

The first objection is to the effect that in giving the truth conditions of predication, it is the members of $H$, not of $D$, which are deployed. Hence the "ontological commitment" of the semantics is to these, and these are 
precise. ${ }^{25}$ In reply to this, two points are relevant. First, the fact that $P a$ is true in a precisification iff the precisification of $a$ is in the precisification of $P$ there, in no way shows that $a$ itself is not real. Indeed, the precisifications are precisifications of something, namely, a. Note that the quantifiers in our language range over the objects in $D$, not $H$. Secondly, even if the only ontologically real things were the members of $H$, it does not follow that the world contains no vague objects. The construction is neutral with respect to the question of whether the precisifications are themselves completely precise. Thus, one might think that the shoreline of mainland Australia provides a precisification of New Holland. But that boundary is itself vague, due to tides, etc. One may take the denizens of $H$ to be completely precise, subject to no further precisification; but nothing in the construction requires this. Indeed, there is no reason why $H$ should not be a subset of $D$, so that its members may themselves have more than one precisification.

The second objection takes off from a paper by David Lewis which comments on Evans' argument. ${ }^{26}$ There, he suggests a way of interpreting the argument as a defence of the view that there are no vague objects. Lewis, too, balks at the application of SI, but for a quite different reason. Names may refer to different things in different precisifications. They are not, then, rigid designators. As is well known, SI fails for non-rigid designators in modal contexts. ${ }^{27}$ Thus, let $n$ be an abbreviation of 'the number of planets'. Then it may be true that $n=8$, and that $\square 8=8$. It is not true that $\square 8=n$. Now, if this is what is going on in Evans' argument, ${ }^{28}$ it means that names are being precisified, not objects. Hence, vagueness is simply in language, not in the objects that the language refers to.

One might suppose that one can object to my account of vague objects in re by making the same point. After all, one might think, given a name, what it refers to is different at every world, so it is changing its denotation. This is not correct, though. For the names in the language are rigid: at every world, they refer to exactly the same things: members of $D$. (That is, for names, $\delta$ is not world-relativised.) The difference between rigid and non-rigid designators shows up in their logic. ${ }^{29}$ Thus, if $a$ is not a rigid designator, the inference $\square P a \vdash \exists x \square P x$ is invalid. The fact that at every

\footnotetext{
${ }^{25}$ Max Cresswell suggested to me that one may see some of Quine's objections to Carnap's notion of intentional objects - which are, similarly, functions from worlds to objects - in this way.

${ }^{26}$ Lewis (1988).

${ }^{27}$ See, e.g., Priest (2008), 16.4 .

${ }^{28}$ Burgess (1989) argues very plausibly that it is not.

${ }^{29}$ See Priest (2008), 17.2.13.
} 
world the denotation of ' $a$ ' has the property $P$ does not show that there is some one thing that does so at all worlds: the denotation of ' $a$ ' may change from world to world. By contrast, as it easy to check, this inference is perfectly valid in the semantics of $\S 3$. Finally, and in any case, though the precisification function, $\pi$, may induce a precisification of names, it is a function on objects (members of $D$ ), not names. ${ }^{30}$

\section{Conclusion}

What we have seen, then, is that the semantics give us a way of understanding vague objects. Their vagueness is constituted precisely by the fact that they can be precisified in different ways. (One could, after all, precisify Mt Everest by constructing any number of different fences around it.) And the semantics highlights a central failing of Evans' argument, to boot: substitutivity in modal contexts. ${ }^{31}$

\section{References}

[1] Burgess, J. (1989), 'Evans Misrepresented', Analysis 49: 112-119.

[2] Evans, G. (1978), 'Can There Be Vague Objects?', Analysis 38: 208; reprinted as ch. 17 of Keefe and Smith (1996).

[3] Field, H. (2003), 'No Fact of the Matter', Australasian Journal of Philosophy 81: 457-480.

[4] Keefe, R., and Smith P. (eds.) (1996), Vagueness: a Reader, Cambridge, MA: MIT Press.

[5] Kripke, S. (1971), 'Identity and Necessity', pp. 135-64 of M. Munitz (ed.), Identity and Individuation, New York, NY: NYU Press; reprinted

\footnotetext{
${ }^{30}$ Compare how precisification works with respect to predicates in these semantics. See fn 18.

${ }^{31}$ Versions of this paper were given as talks at a meeting of the Australasian Association for Logic (Adelaide, July 2017), Syracuse University (September 2017), Princeton University (April 2018), University of St Andrews (April 2018), the National Autonomous University of Mexico (September 2018), and the conference ManyVal 2019, Universityof Bucharest (November 2019). I am grateful to the members of the audiences for helpful comments, and especially to Axel Barteló, JC Beall, Aaron Cotnoir, Max Cresswell, André Gallois, Katherine Hawley, Lloyd Humberstone, Bruno Jacinto, Mark Johnston, Boris Kment, Harvey Lederman, Daniel Nolan, and Stephen Read. Finally, thanks go to an anonymous referee of the journal.
}

Australasian Journal of Logic (18:3) 2021, Article no. 1 
as ch. 2 of S. P. Schwartz (ed.), Naming, Necessity and Natural Kinds, Ithaca, NY: Cornell University Press.

[6] Lewis, D. (1988), 'Vague Identity: Evans Misunderstood', Analysis 48: 128-130; reprinted as ch. 18 of Keefe and Smith (1996).

[7] Priest, G. (2002), 'Paraconsistent Logic', pp. 287-393, vol. 6, of D. Gabbay and F. Guenthner (eds.), Handbook of Philosophical Logic, 2nd edn, Dordrecht: Kluwer Academic Publishers.

[8] Priest, G. (2008), Introduction to Non-Classical Logic: From If to Is, Cambridge: Cambridge University Press.

[9] Tye, M. (1994), 'Sorites Paradoxes and the Semantics of Vagueness', Philosophical Perspectives 8: 189-206; reprinted as ch. 15 of Keefe and Smith (1996).

Australasian Journal of Logic (18:3) 2021, Article no. 1 